\title{
DIGITALCOMMONS
}

$11-1-2003$

\section{End Matter}

JMASM Editors

Follow this and additional works at: http:// digitalcommons.wayne.edu/jmasm

\section{Recommended Citation}

Editors, JMASM (2003) "End Matter," Journal of Modern Applied Statistical Methods: Vol. 2 : Iss. 2 , Article 29. DOI: $10.22237 /$ jmasm/1067646540

Available at: http://digitalcommons.wayne.edu/jmasm/vol2/iss2/29 


\section{NEW IN 2004}

\section{significance \\ statistics making sense \\ The new magazine of the Royal Statistical Society}

\section{Edited by Helen Joyce}

Significance is a new quarterly magazine for anyone interested in statistics and the analysis and interpretation of data. It aims to communicate and demonstrate, in an entertaining and thought-provoking way, the practical use of statistics in all walks of life and to show how statistics benefit society.

Articles are largely non-technical and hence accessible and appealing, not only to members of the profession, but to all users of statistics.

As well as promoting the discipline and covering topics of professional relevance, Significance contains a mixture of statistics in the news, casestudies, reviews of existing and newly developing areas of statistics, the application of techniques in practice and problem solving, all with an international flavour.

\section{Special Introductory Offer:}

$25 \%$ discount on a new personal subscription Plus Great Discounts for Students !

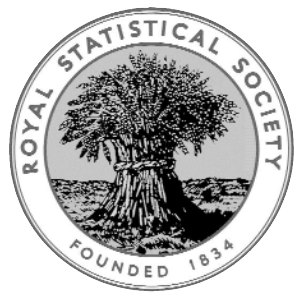




\section{PASS 2002}

\section{Power Analysis and Sample Size Software from NCSS}

PASS performs power analysis and calculates sample sizes. Use it before you begin a study to calculate an appropriate sample size (it meets the requirements of government agencies that want technical justification of the sample size you have used). Use it after a study to determine if your sample size was large enough. PASS calculates the sample sizes necessary to perform all of the statistical tests listed below.

A power analysis usually involves several "what if" questions. PASS lets you solve for power, sample size, effect size, and alpha level. It automatically creates appropriate tables and charts of the results.

$\boldsymbol{P A S S}$ is accurate. It has been extensively verified using books and reference articles. Proof of the accuracy of each procedure is included in the extensive documentation.

PASS is a standalone system. Although it is integrated with NCSS, you do not have to own NCSS to run it. You can use it with any statistical software you want.

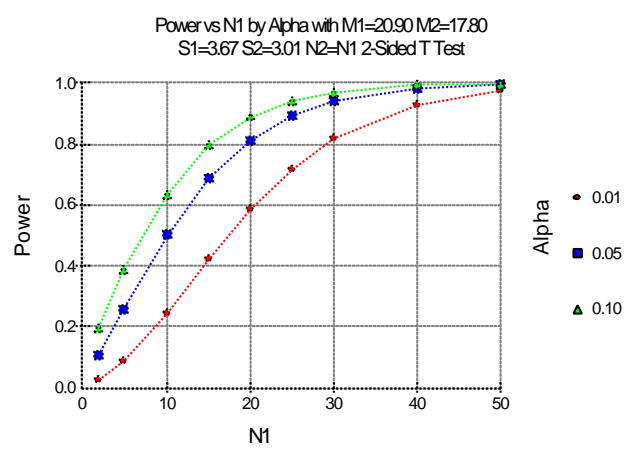

PASS comes with two manuals that contain tutorials, examples, annotated output, references, formulas, verification, and complete instructions on each procedure. And, if you cannot find an answer in the manual, our free technical support staff (which includes a $\mathrm{PhD}$ statistician) is available.

\section{System Requirements}

PASS runs on Windows 95/98/ME/NT/ 2000/XP with at least 32 megs of RAM and 30 megs of hard disk space.

$\boldsymbol{P A S S}$ sells for as little as $\mathbf{\$ 4 4 9 . 9 5}$.
PASS Beats the Competition!

No other program calculates sample sizes and power for as many different statistical procedures as does PASS.

Specifying your input is easy, especially with the online help and manual.

PASS automatically displays charts and graphs along with numeric tables and text summaries in a portable format that is cut and paste compatible with all word processors so you can easily include the results in your proposal.

Choose PASS. It's more comprehensive, easier-to-use, accurate, and less expensive than any other sample size program on the market.

\section{Trial Copy Available}

You can try out $\boldsymbol{P A S S}$ by downloading it from our website. This trial copy is good for 30 days. We are sure you will agree that it is the easiest and most comprehensive power analysis and sample size program available.
Analysis of Variance

Factorial AOV

Fixed Effects AOV

Geisser-Greenhouse

MANOVA*

Multiple Comparisons*

One-Way AOV

Planned Comparisons

Randomized Block AOV

New Repeated Measures AOV*

Regression / Correlation

Correlations (one or two)

Cox Regression*

Logistic Regression

Multiple Regression

Poisson Regression*

Intraclass Correlation

Linear Regression

\section{Proportions}

Chi-Square Test

Confidence Interval

Equivalence of McNemar*

Equivalence of Proportions

Fisher's Exact Test

Group Sequential Proportions

Matched Case-Control

McNemar Test

Odds Ratio Estimator

One-Stage Designs*

Proportions -1 or 2

Two Stage Designs (Simon's)

Three-Stage Designs*

Miscellaneous Tests

Exponential Means -1 or $2^{*}$

ROC Curves -1 or $2^{*}$

Variances -1 or 2

\section{T Tests}

Cluster Randomization

Confidence Intervals

Equivalence T Tests

Hotelling's T-Squared*

Group Sequential T Tests

Mann-Whitney Test

One-Sample T-Tests

Paired T-Tests

Standard Deviation Estimator

Two-Sample T-Tests

Wilcoxon Test

\section{Survival Analysis}

Cox Regression*

Logrank Survival -Simple

Logrank Survival - Advanced*

Group Sequential - Survival

Post-Marketing Surveillance

ROC Curves -1 or $2^{*}$
Group Sequential Tests

Alpha Spending Functions

Lan-DeMets Approach

Means

Proportions

Survival Curves

Equivalence

Means

Proportions

Correlated Proportions*

Miscellaneous Features

Automatic Graphics

Finite Population Corrections

Solves for any parameter

Text Summary

Unequal N's

*New in PASS 2002 


\section{WHAT'S NEW IN PASS 2002?}

Thirteen new procedures have been added to $\boldsymbol{P A S S}$ as well as a new home-base window and a new Guide Me facility.

\section{MANY NEW PROCEDURES}

The new procedures include a new multifactor repeated measures program that includes multivariate tests, Cox proportional hazards regression, Poisson regression, MANOVA, equivalence testing when proportions are correlated, multiple comparisons, ROC curves, and Hotelling's T-squared.

\section{TEXT STATEMENTS}

The text output translates the numeric output into easy-to-understand sentences. These statements may be transferred directly into your grant proposals and reports.

\section{GRAPHICS}

The creation of charts and graphs is easy in PASS. These charts are easily transferred into other programs such as MS PowerPoint and MS Word.

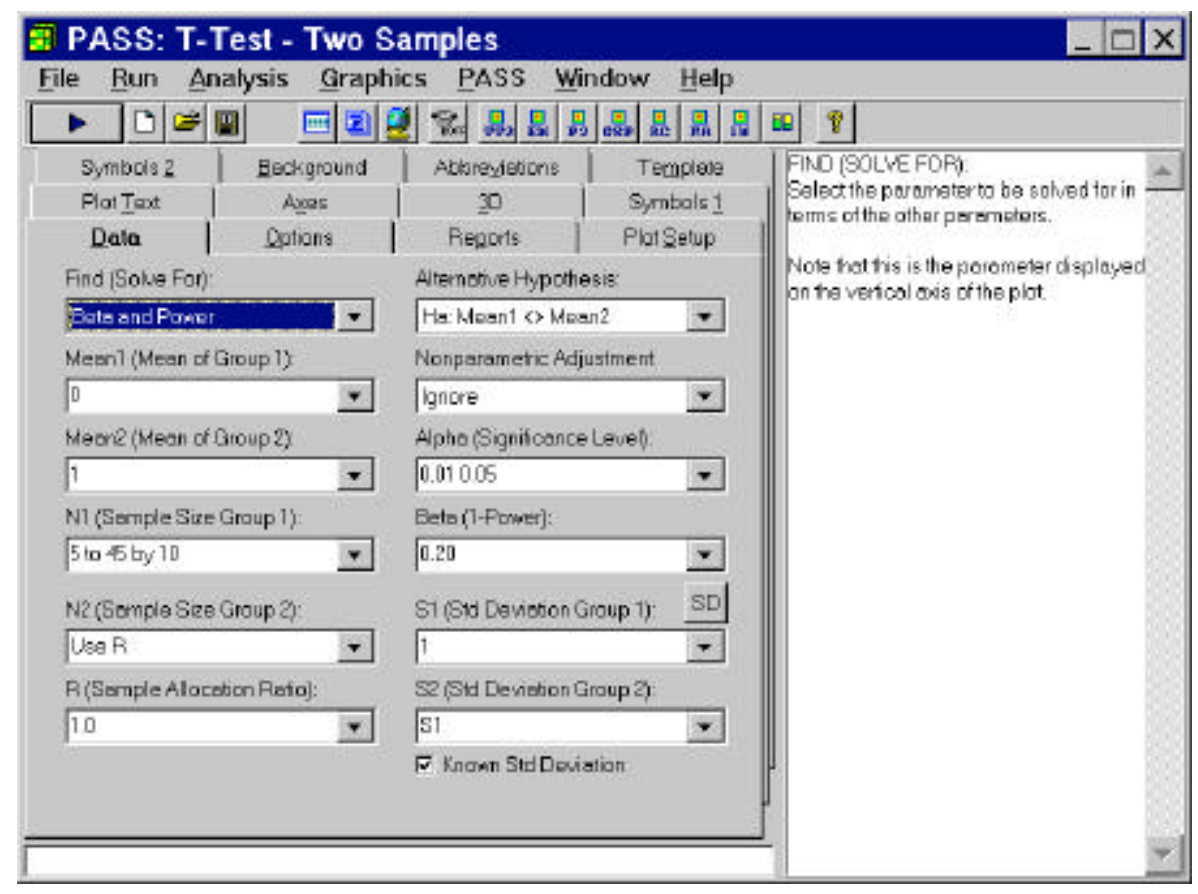

\section{NEW USER'S GUIDE II}

A new, 250-page manual describes each new procedure in detail. Each chapter contains explanations, formulas, examples, and accuracy verification.

The complete manual is stored in PDF format on the CD so that you can read and printout your own copy.

GUIDE ME

The new Guide Me facility makes it easy for first time users to enter parameter values.

The program literally steps you through those options that are necessary for the sample size calculation.

\section{NEW HOME BASE}

A new home base window has been added just for PASS users. This window helps you select the appropriate program module.

\section{COX REGRESSION}

A new Cox regression procedure has been added to perform power analysis and sample size calculation for this important statistical technique.

\section{REPEATED MEASURES}

A new repeated-measures analysis module has been added that lets you analyze designs with up to three grouping factors and up to three repeated factors. The analysis includes both the univariate $\mathrm{F}$ test and three common multivariate tests including Wilks Lambda.

\section{RECENT REVIEW}

In a recent review, 17 of 19 reviewers selected $\boldsymbol{P A S S}$ as the program they would recommend to their colleagues.

\section{PASS calculates sample sizes for...}

Please rush me my own personal license of PASS 2002.

Qty

PASS 2002 Deluxe (CD and User's Guide): \$499.95.............

PASS 2002 CD (electronic documentation): \$449.95 .........\$ $\$$

PASS 2002 5-User Pack (CD \& 5 licenses): \$1495.00........\$

PASS 2002 25-User Pack (CD \& 25 licenses): \$3995.00 ....\$

PASS 2002 User's Guide II (printed manual): $\$ 30.00 \ldots \ldots \ldots . .$.

PASS 2002 Upgrade CD for PASS 2000 users: \$149.95 ......\$

Typical Shipping \& Handling: USA: \$9 regular, \$22 2-day, \$33

overnight. Canada: \$19 Mail. Europe: \$50 Fedex......................\$

Total:

$\$$

FOR FASTEST DELIVERY, ORDER ONLINE AT WWW.NCSS.COM

Email your order to sales@ncss.com

Fax your order to (801) 546-3907

NCSS, 329 North 1000 East, Kaysville, UT 84037

(800) 898-6109 or (801) 546-0445
My Payment Options:

Check enclosed

___ Please charge my: ___VISA __MasterCard __Amex

Purchase order enclosed

Card Number Expires

Signature

Please provide daytime phone:

Ship my PASS 2002 to:

NAME

COMPANY

ADDRESS

CITY/STATE/ZIP

COUNTRY (IF OTHER THAN U.S.) 
NCSS

329 North 1000 East

Kaysville, Utah 84037

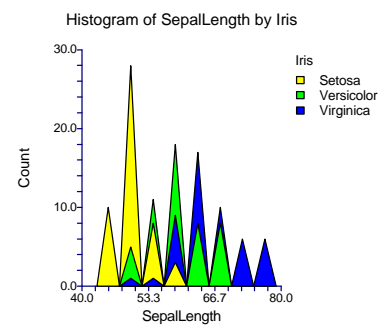

\section{Announcing NCSS 2004 Seventeen New Procedures}

NCSS 2004

is a new edition of our popular statistical NCSS package that adds seventeen new procedures.

\section{New Procedures}

Two Independent Proportions

Two Correlated Proportions

One-Sample Binary Diagnostic Tests

Two-Sample Binary Diagnostic Tests

Paired-Sample Binary Diagnostic Tests

Cluster Sample Binary Diagnostic Tests

Meta-Analysis of Proportions

Meta-Analysis of Correlated Proportions

Meta-Analysis of Means

Meta-Analysis of Hazard Ratios

Curve Fitting

Tolerance Intervals

Comparative Histograms

ROC Curves

Elapsed Time Calculator

T-Test from Means and SD's

Hybrid Appraisal (Feedback) Model

\section{Documentation}

The printed, 330-page manual, called

NCSS User's Guide V, is available for

$\$ 29.95$. An electronic (pdf) version of

the manual is included on the distribution

$\mathrm{CD}$ and in the Help system.

\section{Two Proportions}

Several new exact and asymptotic techniques were added for hypothesis testing (null, noninferiority, equivalence) and calculating confidence intervals for the difference, ratio, and odds ratio. Designs may be independent or paired. Methods include: Farrington \& Manning, Gart \& Nam, Conditional \& Unconditional Exact, Wilson's Score, Miettinen \& Nurminen, and Chen.

\section{Meta-Analysis}

Procedures for combining studies measuring paired proportions, means, independent proportions, and hazard ratios are available. Plots include the forest plot, radial plot, and L'Abbe plot. Both fixed and random effects models are available for combining the results.

\section{Curve Fitting}

This procedure combines several of our curve fitting programs into one module. It adds many new models such as Michaelis-Menten. It analyzes curves from several groups. It compares fitted models across groups using computerintensive randomization tests. It computes bootstrap confidence intervals.

\section{Tolerance Intervals}

This procedure calculates one and two sided tolerance intervals using both distribution-free (nonparametric) methods and normal distribution (parametric) methods. Tolerance intervals are bounds between which a given percentage of a population falls.

\section{Comparative Histogram}

This procedure displays a comparative histogram created by interspersing or overlaying the individual histograms of two or more groups or variables. This allows the direct comparison of the distributions of several groups.

\section{Random Number Generator} Matsumoto's Mersenne Twister random number generator (cycle length > $10 * * 6000)$ has been implemented.

\section{Binary Diagnostic Tests}

Four new procedures provide the specialized analysis necessary for diagnostic testing with binary outcome data. These provide appropriate specificity and sensitivity output. Four experimental designs can be analyzed including independent or paired groups, comparison with a gold standard, and cluster randomized.

\section{ROC Curves}

This procedure generates both binormal and empirical (nonparametric) ROC curves. It computes comparative measures such as the whole, and partial, area under the ROC curve. It provides statistical tests comparing the AUC's and partial AUC's for paired and independent sample designs.

\section{Hybrid (Feedback) Model}

This new edition of our hybrid appraisal model fitting program includes several new optimization methods for calibrating parameters including a new genetic algorithm. Model specification is easier. Binary variables are automatically generated from class variables.

\section{Statistical Innovations Products}

Through a special arrangement with Statistical Innovations (S.I.), NCSS customers will receive $\$ 100$ discounts on:

Latent GOLD $®$ - latent class modeling

SI-CHAID ${ }^{\circledR}$ - segmentation trees

GOLDMineR ${ }^{\circledR}$ - ordinal regression

For demos and other info visit: www.statisticalinnovations.com 
Please rush me the following products:

Qty

NCSS 2004 CD upgrade from NCSS 2001, \$149.95 $\$$

NCSS 2004 User's Guide V, \$29.95 $\$$

NCSS 2004 CD, upgrade from earlier versions, \$249.95 .......... \$

NCSS 2004 Deluxe (CD and Printed Manuals), \$599.95 ……... \$

PASS 2002 Deluxe, $\$ 499.95$ ..\$

Latent Gold ${ }^{\circledR}$ from S.I., $\$ 995-\$ 100$ NCSS Discount $=\$ 895 \ldots . . . . \$$

GoldMineR® from S.I., $\$ 695-\$ 100$ NCSS Discount $=\$ 595$..... $\$$

CHAID $®$ Plus from S.I., $\$ 695-\$ 100$ NCSS Discount $=\$ 595 \ldots . \$$

Approximate shipping--depends on which manuals are ordered (U.S: $\$ 10$ ground, \$18 2-day, or \$33 overnight) (Canada \$24) (All other countries $\$ 10$ ) (Add $\$ 5$ U.S. or $\$ 40$ International for any S.I. product) ........ \$

Total...........

CALL: (800) 898-6109 FAX: (801) 546-3907

ONLINE: www.ncss.com

MAIL: NCSS, 329 North 1000 East, Kaysville, UT 84037

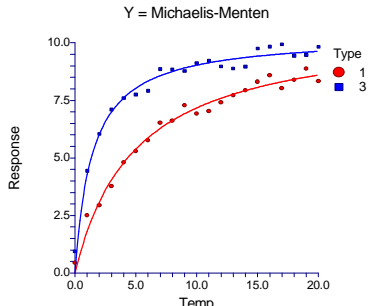

ROC Curve of Fever

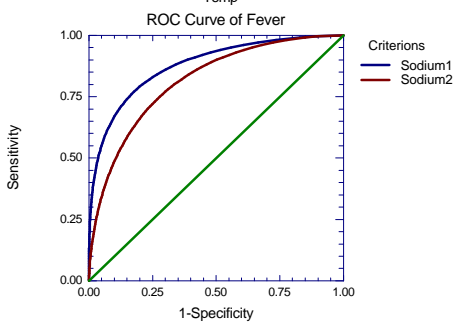

Histogram of Sepallength by lirs

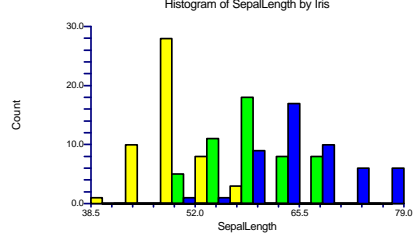

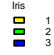

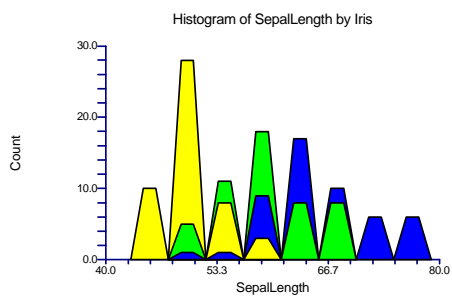

My Payment Option:

Check enclosed

Please charge my: _ VISA _ MasterCard

Amex Purchase order attached

Card Number Exp

Signature

Telephone:

Email:

Ship to:

NAME

ADDRESS

ADDRESS

ADDRESS

CITY STATE

ZIP/POSTAL CODE COUNTRY

\section{Statistical and Graphics Procedures Available in NCSS 2004}

Analysis of Variance / T-Tests Analysis of Covariance

Analysis of Variance

Barlett Variance Test Crossover Design Analysis

Factorial Design Analysis

Friedman Test

Geiser-Greenhouse Correction

General Linear Models

Mann-Whitney Test

MANOVA

Multiple Comparison Tests

One-Way ANOVA

Paired T-Tests

Power Calculations

Repeated Measures ANOVA

T-Tests - One or Two Groups

T-Tests - From Means \& SD's

Wilcoxon Test

Time Series Analysis

ARIMA / Box - Jenkins

Decomposition

Exponential Smoothing

Harmonic Analysis

Holt - Winters

Seasonal Analysis

Spectral Analysis

Trend Analysis

*New Edition in 2004
Regression / Correlation Survival / Reliability

Experimental Designs

Balanced Inc. Block

Box-Behnken

Central Composite

D-Optimal Designs

Fractional Factoria

Latin Squares

Placket-Burman

Response Surface

Screening

Taguchi All-Possible Search Canonical Correlation Correlation Matrices Cox Regression

Kendall's Tau Correlation Linear Regression Logistic Regression Multiple Regression Nonlinear Regression PC Regression Poisson Regression Response-Surface Ridge Regression Robust Regression Stepwise Regression Spearman Correlation Variable Selection

Accelerated Life Tests

Cox Regression

Cumulative Incidence Exponential Fitting

Extreme-Value Fitting

Hazard Rates

Kaplan-Meier Curves

Life-Table Analysis

Lognormal Fitting

Log-Rank Tests

Probit Analysis

Proportional-Hazards

Reliability Analysis

Survival Distributions

Time Calculator*

Weibull Analysis

Quality Control

Xbar-R Chart

$C, P, N P, U$ Charts

Capability Analysis

Cusum, EWMA Chart

Individuals Chart

Moving Average Chart

Pareto Chart

R \& R Studies

Cluster Analysis

Discriminant Analysis

Factor Analysis

Hotelling's T-Squared

Item Analysis

Loglinear Models
Curve Fitting

Bootstrap C.I.'s*

Built-In Models

Group Fitting and Testing

Model Searching

Nonlinear Regression

Randomization Tests*

Ratio of Polynomials

User-Specified Models

Miscellaneous

Area Under Curve

Bootstrapping

Chi-Square Test

Confidence Limits

Cross Tabulation

Data Screening

Fisher's Exact Test

Frequency Distributions

Mantel-Haenszel Test

Correspondence Analysis

Item Response Analysis

MANOVA

Multi-Way Tables

Multidimensional Scaling

Principal Components
Meta-Analysis* Independent Proportions* Correlated Proportions* Hazard Ratios* Means*

Binary Diagnostic Tests* One Sample*

Two Samples* Paired Samples Clustered Samples*

Proportions Tolerance Intervals* Two Independent ${ }^{\star}$

Two Correlated*

Exact Tests*

Exact Confidence Intervals ${ }^{\star}$

Farrington-Manning ${ }^{\star}$

Fisher Exact Test

Gart-Nam* Method

McNemar Test

Miettinen-Nurminen* Wilson's Score* Method Equivalence Tests* Noninferiority Tests*

Mass Appraisa Comparables Reports Hybrid (Feedback) Model ${ }^{\star}$ Nonlinear Regression Sales Ratios 


\section{Qualitative research has come a long way...}

from this...

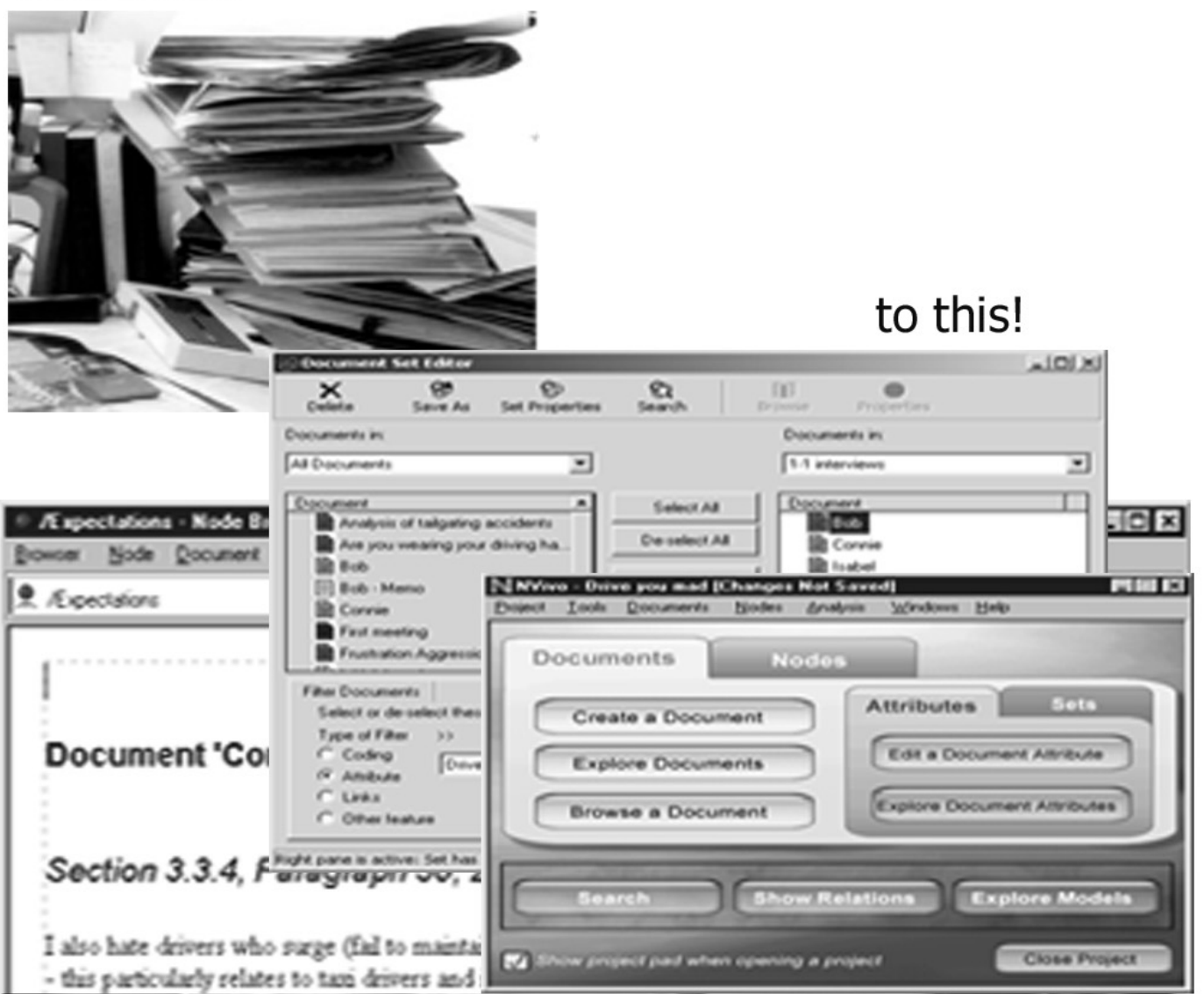

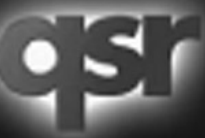

THE LATEST PRODUCTS HAVE ARRIVED www.qsinternational.com
Ne

ave

WORLD LEADING PRCOUCTS FROM THE NUO'IST LINE OF SOFTMARE

Read more about QSR software in this edition of JMASM. 
"Perfection is achieved, not when there is nothing more to add, but when there is nothing left to take away."

- Antoine de Saint Exupery

F is a carefully crafted subset of the most recent version of Fortran, the world's most powerful numeric language.

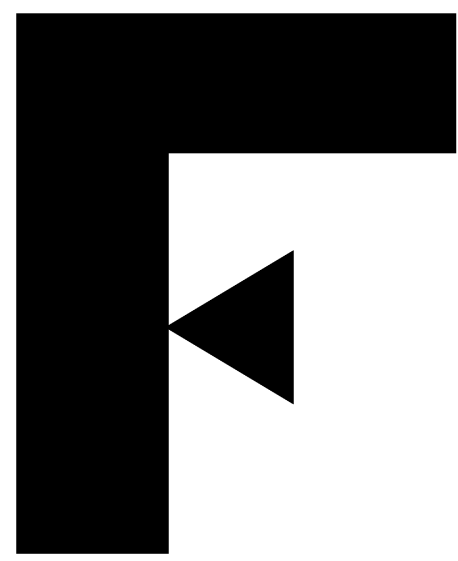

Using F has some very significant advantages:

- Programs written in F will compile with any Fortran compiler

- $\mathrm{F}$ is easier to use than other popular programming languages

- F compilers are free and available for Linux, Windows, and Solaris

- Several books on F are available

- F programs may be linked with C, Fortran 95, or older Fortran 77 programs

F retains the modern features of Fortran-modules and data abstraction, for example-but discards older error-prone facilities of Fortran.

It is a safe and portable programming language.

F encourages Module-Oriented Programming.

It is ideal for teaching a programming language in science, engineering, mathematics, and finance.

It is ideal for new numerically intensive programs.

The Fortran Company

11155 E. Mountain Gate Place, Tucson, AZ 85749 USA $+1-520-256-1455+1-520-760-1397$ (fax) http://www.fortran.com info@fortran.com 


\section{GGUM2000}

\section{Item Response Theory Models for Unfolding}

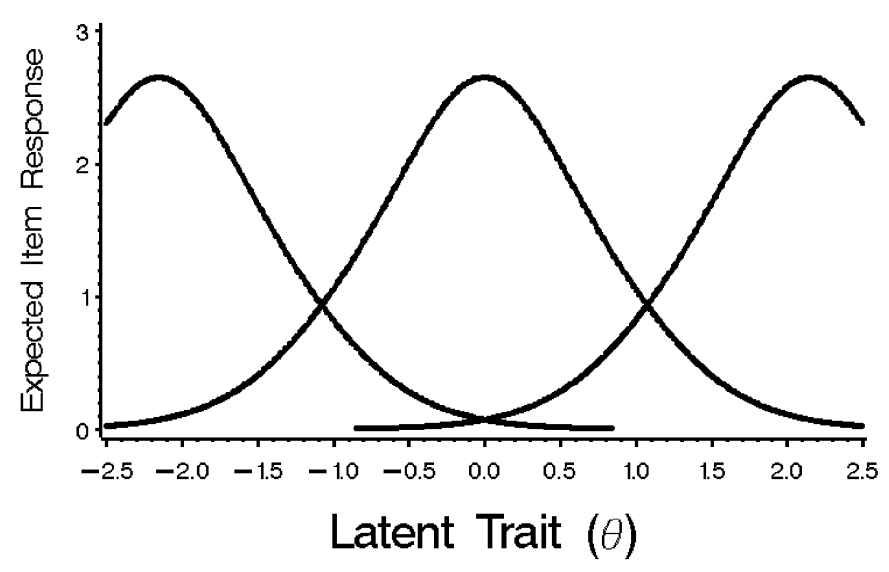

The GGUM2000 software system estimates parameters in a family of item response theory (IRT) models that unfold polytomous responses to questionnaire items. These models assume that persons and items can be jointly represented as locations on a latent unidimensional continuum. A single-peaked, nonmonotonic response function is the key feature that distinguishes unfolding IRT models from traditional, "cumulative" IRT models. This response function suggests that a higher item score is more likely to the extent that an individual is located close to a given item on the underlying continuum. Such single-peaked functions are appropriate in many situations including attitude measurement with Likert or Thurstone scales, and preference measurement with stimulus rating scales. This family of models can also be used to determine the locations of respondents in particular developmental processes that occur in stages.

The GGUM2000 system estimates item parameters using marginal maximum likelihood, and person parameters are estimated using an expected a posteriori (EAP) technique. The program allows for up to 100 items with 2-10 response categories per item, and up to 2000 respondents. The software is accompanied by a detailed user's manual. GGUM2000 is free and can be downloaded from:

\section{http://www.education.umd.edu/EDMS/tutorials}

Start putting the power of unfolding IRT models to work in your attitude and preference measurement endeavors. Download your free copy of GGUM2000 today! 


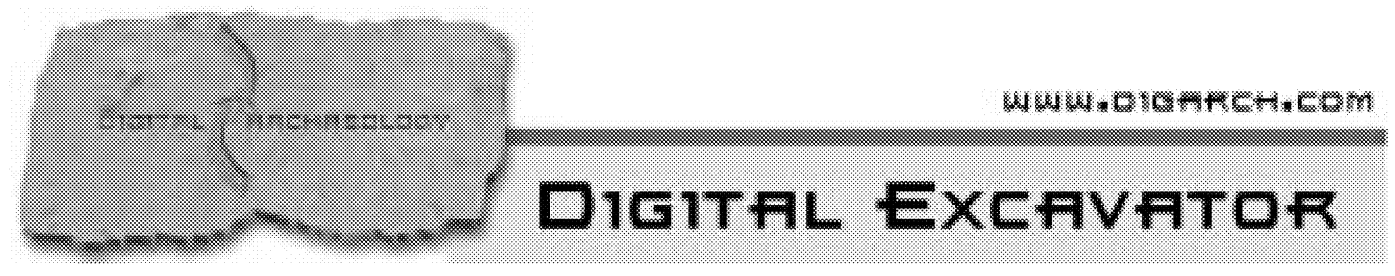

Are you involved in Data Modeling or Data Mining?

\section{Are you spending a large percentage of your time dealing with data issues?}

If so, you will be happy to know that we have developed a tool that specifically addresses the data prep tasks associated with data modeling and data mining. The tool is called the Digital Excavator from Digital Archaeology (www.digarch.com). Data modelers are well aware of the time-consuming and sometimes frustrating nature of data set-up. In many cases data preparation can represent $60 \%$ $80 \%$ of the data mining project length. With Digital Archaeology's Digital Excavator, data preparation tasks are streamlined, results are more accurate, and the modeler has more time to focus on finding the appropriate mathematical solution--rather than wasting time with painful data issues. Digital Archaeology's software is intuitive, visual, self-documenting, and deploys what a number of analysts and customers have termed the "most elegant" user interface for data analysis and exploration ever conceived. It's the only tool specifically designed for the data prep tasks of data modeling.

\section{Visit our website and see for yourself! $\quad$ >>> www.digarch.com}

Functions have been created which perform the following:

- Frequency Distributions

- Categorical Variable Profile

- Continuous Variable Profile

- Histograms

- De-duping

- $\quad$ Find and Replace Missing Values

- $\quad$ Find and Split Out Outliers

- Binning

- Correlation Matrix

- Cross-Tabs

- $\quad$ Panel Variables (Occupancy Map)

- Lag functions

- Decimal Scaling

- $\quad$ Rank and Sample Variables

- $\quad$ Recency, Frequency, Monetary Analysis

- N-Tile Distributions

- Gains Charts

- Many others
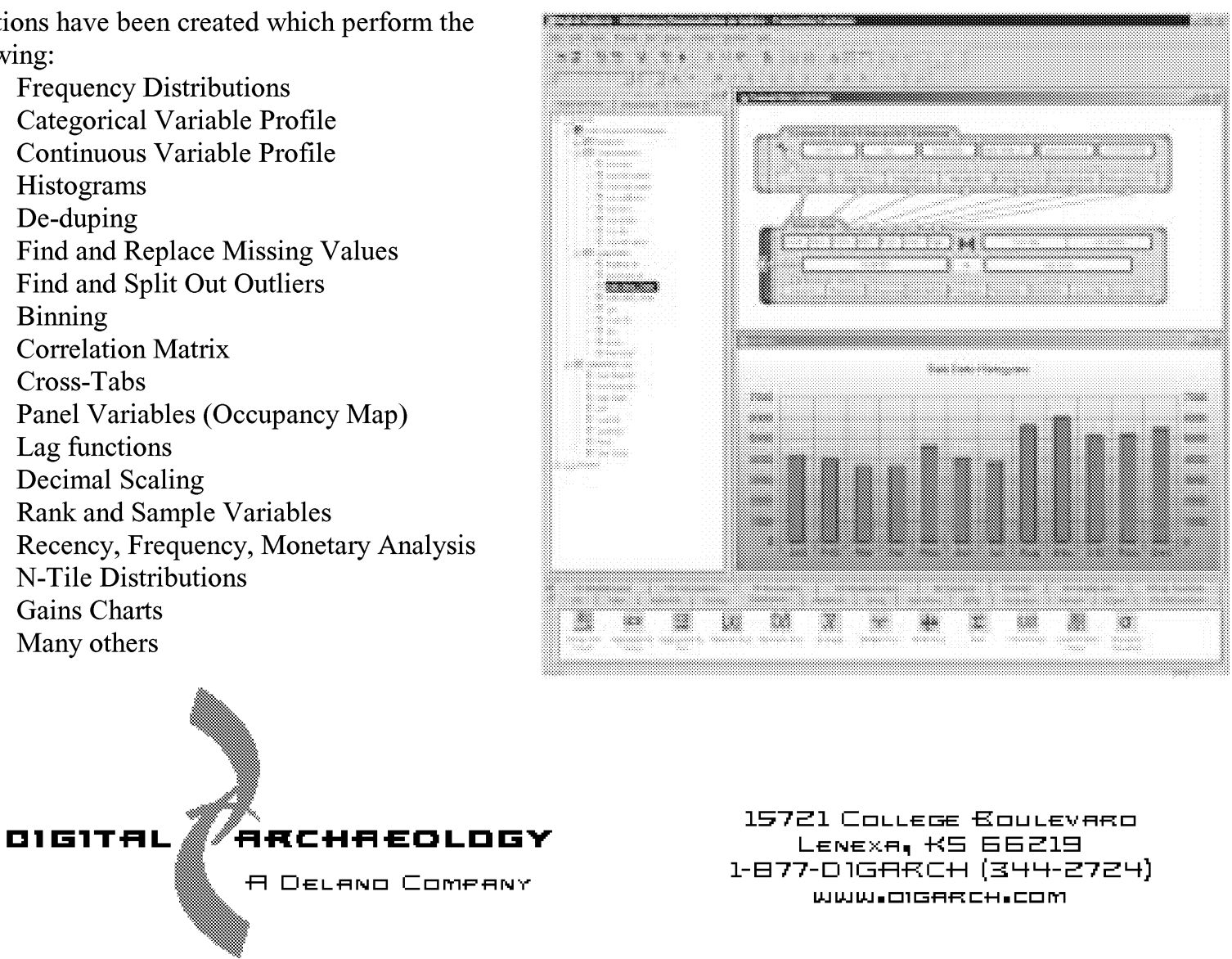


\section{Numerical Recipes in Fortran from Cambridge University Press}

\section{Numerical Recipes in Fortran 77 \\ Volume 1 of Fortran Numerical Recipes \\ Second Edition \\ William H. Press, Saul A. Teukolsky, William T.Vetterling, and Brian P. Flannery}

"This reviewer knows of no other single source of so much material of this nature. Highly recommended."

$$
\text { - Choice }
$$

"... a valuable resource for those with a specific need for numerical software. The routines are prefaced with lucid, selfcontained explanations....highly recommended for those who require the use and understanding of numerical software."

-SIAM Review

1992992 pp. 0-521-43064-X Hardback $\$ 70.00$

\section{Highlights include:}

- A chapter on integral equations and inverse methods

4. Multigrid and other methods for solving partial differential equations

9. Improved random namber routines

- Wavelet transforms

- The statistical bootstrap method

- A chapter on "less numerical" algorithms including compression coding and arbitrary precision arithmetic:

\section{Numerical Recipes in Fortran 77 Example Book}

Second Edition

William T.Vetterling, Saul A. Teukolsky, William H. Press, and Brian P. Flannery

$1992 \quad 256 \mathrm{pp} . \quad 0-521-43721-0 \quad$ Paperback $\$ 35.00$

\section{Numerical Recipes in Fortran 90}

The Art of Parallel Scientific Computing

Volume 2 of Fortran Numerical Recipes

Second Edition

\section{William H. Press, Saul A. Teukolsky, William T.Vetterling, and Brian P. Flannery}

"This present volume will contribute decisively to a significant breakthrough, as it provides models not only of the numerical algorithms for which previous editions are already famed, but also of an excellent Fortran 90 style."

-From the Foreword by Michael Metcalf, one of Fortran 90 's original designers and author of FORTRAN 90 Explained

"This book is a classic and is essential reading for anyone concerned with the future of numerical calculation. It is beatifully produced, inexpensive for its content, and a must for any serious worker or student."

- Computing Reviews

Contains a detailed introduction to the Fortran 90 language and to the basic concepts of parallel programming, plus source code for all routines from the second edition of Numerical Recipes.

1996576 pp. 0-521-57439-0 Hardback $\$ 50.00$

\section{Numerical Recipes Multi-Language Code CDROM with LINUX or UNIX Single Screen License}

Source Code for Numerical Recipes in C, C++, Fortran 77, Fortran 90, Pascal, BASIC, Lisp and Modula 2 plus many extras

$2002 \quad 0.521-75036 \sim 9 \quad$ CD $R O M \quad \$ 150.00$

\section{Numerical Recipes Multi-Language Code CDROM with Windows, DOS, or Macintosh Single Screen License}

Source Code for Numerical Recipes in $\mathrm{C}, \mathrm{C}++$, Fortran 77 , Fortran 90, Pascal, BASIC, Lisp and Modula 2 plus many extras

$2002 \quad 0-521-75035-0 \quad$ CD-ROM $\$ 90.00$

Visit us.cambridge.org/numericalrecipes for more information on the complete line of Numerical Recipes products. 


\section{IMPORT XML-FORMATTED DATA INTO SAS EXPORT SAS DATA AS XML-FORMATTED DATA}

Now, an easy way to move CDISC and other XML-formatted data into or out of your SAS-based systems. You don't have to know perl, XSLT, Xpath, Java ${ }^{\circledR}$, or exotic languages. The remarkable Tekoa ${ }^{\text {sm }} \mathrm{XML}$ plug-in does it all for you.

Provided free of charge to any SAS user currently wrestling with XML. Developed by Zurich Biostatistics, the pioneer in SAS/XML integration.

\section{FREE. EASY. AND IT WORKS.}

Just e-mail Michael Palmer (mcpalmer@zbi.net) and receive the fully-functional, proven Tekoa XML plug-in by e-mail.

No charge. No obligation. No hassle. (We even support the tool. Imagine.)

\section{Zurich Biostatistics, Inc.}

45 Park Place South, PMB 178, Morristown, NJ 07960973 727-0025 wmw.zbi.net

\section{$X M L$ is easy if you know how. And we do.}

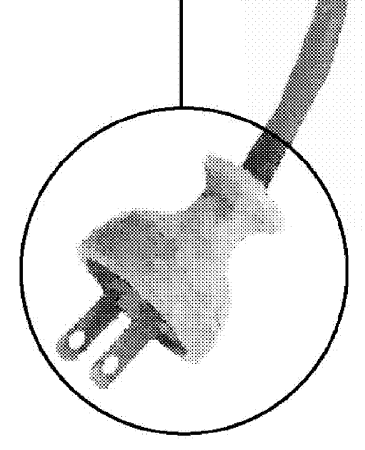

Tekoa XML Technology is a service mark of Zurich Biostatistics. In SAS is a registered trademark of SAS Institute Inc Java is a registered trademark of Sun Microsystems, Inc.

\section{Resampling Stats for Excel}

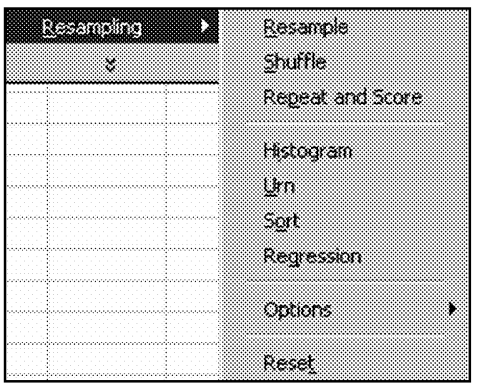

Select the data you want to resample, select "resample" or "shuffle," then specify an output range for the resampled data. Calculate a statistic of interest, select "Repeat \& Score," and the resampling operation will be repeated thousands (or tens of thousands) of times, and each time the value of your statistic of interest will be recorded. Does not use Excel's random number generator.

View complete user guide and download free 30-day trial at <www.resample.com>

\$249 commercial •\$149 personal/academic • \$89 student

612 N Jackson St., Arlington, VA 22201 Tel 703-522-2713 • Fax 703-522-5846 stats@resample.com

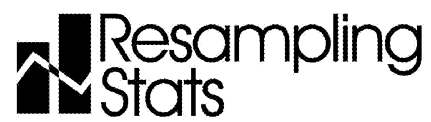




\section{JOIN DIVISION 5 OF APA!}

The Division of Evaluation, Measurement, and Statistics of the American Psychological Association draws together individuals whose professional activities and/or interests include assessment, evaluation, measurement, and statistics. The disciplinary affiliation of division membership reaches well beyond psychology, includes both members and non-members of APA, and welcomes graduate students.

Benefits of membership include:

- $\quad$ subscription to Psychological Methods or Psychological Assessment (student members, who pay a reduced fee, do not automatically receive a journal, but may do so for an additional $\$ 18$ )

- $\quad$ The Score - the division's quarterly newsletter

- Division's Listservs, which provide an opportunity for substantive discussions as well as the dissemination of important information (e.g., job openings, grant information, workshops)

Cost of membership: $\$ 38$ (APA membership not required); student membership is only $\$ 8$

For further information, please contact the Division's Membership Chair, Yossef Ben-Porath (ybenpora@kent.edu) or check out the Division's website:

http://www.apa.org/divisions/div5/

\section{ARE YOU INTERESTED IN AN ORGANIZATION DEVOTED TO EDUCATIONAL AND BEHAVIORAL STATISTICS?}

Become a member of the Special Interest Group - Educational Statisticians of the American Educational Research Association (SIG-ES of AERA)!

The mission of SIG-ES is to increase the interaction among educational researchers interested in the theory, applications, and teaching of statistics in the social sciences.

Each Spring, as part of the overall AERA annual meeting, there are seven sessions sponsored by SIG-ES devoted to educational statistics and statistics education.

We also publish a twice-yearly electronic newsletter.

Past issues of the SIG-ES newsletter and other information regarding SIG-ES can be found at http://orme.uark.edu/edstatsig.htm

To join SIG-ES you must be a member of AERA. Dues are $\$ 5.00$ per year.

For more information, contact Joan Garfield, President of the SIG-ES, at jbg@umn.edu. 
Position Available: Top bio-tech company seeks a seasoned statistical manager to hire, develop and lead a team of applied statisticians. Primary role is to integrate statistical methodology and practice into product/process development, manufacturing operations and quality. This key leader will provide linkage between manufacturing, engineering, development and biostatistics. MS in statistics or related field.

Research Statistician: Established clinical group adding staff to provide dedicated preclinical support to a development center. Interact and support scientists with formulation, stability testing, bioanalytics and bio assays. PhD w/3 yrs or MS w/ 6 years industry experience required along with expertise in complicated design methods. Northeast location.

Contact Information: Eve Kriz, Smith Hanley Associates, 99 Park Avenue, New York, NY 10016, 212-687-9696 ext. 228, ekriz@smithhanley.com.

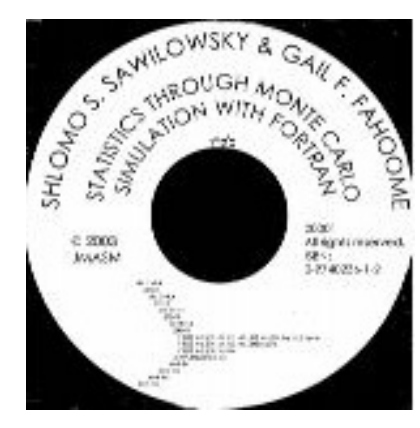

\section{Statistic s Through Monte Carlo Simulation}

\section{With Fortra n}

Shlomo S. Sa wilowsky and Gail F. Fahoome

Copyright $\odot 2003$ ISBN: 0-9740236-0-4

Purchase Email, CD, \& Softcover Versions Online Via Secure Paypal At

http://tbf.coe.wayne.edu/jmasm 


\section{Instructions For Authors}

Follow these guidelines when submitting a manuscript:

1. JMASM uses a modified American Psychological Association style guideline.

2. Submissions are accepted via e-mail only. Send them to the Editorial Assistant at ea@edstat.coe.wayne.edu. Provide name, affiliation, address, e-mail address, and 30 word biographical statements for all authors in the body of the email message.

3. There should be no material identifying authorship except on the title page. A statement should be included in the body of the e-mail that, where applicable, indicating proper human subjects protocols were followed, including informed consent. A statement should be included in the body of the e--mail indicating the manuscript is not under consideration at another journal.

4. Provide the manuscript as an external e-mail attachment in MS Word for the PC format only. (Wordperfect and .rtf formats may be acceptable - please inquire.) Please note that Tex (in its various versions), Exp, and Adobe .pdf formats are designed to produce the final presentation of text. They are not amenable to the editing process, and are not acceptable for manuscript submission.

5. The text maximum is 20 pages double spaced, not including tables, figures, graphs, and references. Use 11 point Times Roman font. If the technical expertise is available, submit the manuscript in two column format.

6. Create tables without boxes or vertical lines. Place tables, figures, and graphs "in-line", not at the end of the manuscript. Figures may be in .jpg, .tif, .png, and other formats readable by Adobe Illustrator or Photoshop.

7. The manuscript should contain an Abstract with a 50 word maximum, following by a list of key words or phrases. Major headings are Introduction, Methodology, Results, Conclusion, and References. Center headings. Subheadings are left justified; capitalize only the first letter of each word. Sub-subheadings are leftjustified, indent optional.

8. Do not use underlining in the manuscript. Do not use bold, except for (a) matrices, or (b) emphasis within a table, figure, or graph. Do not number sections. Number all formulas, tables, figures, and graphs, but do not use italics, bold, or underline. Do not number references. Do not use footnotes or endnotes.

9. In the References section, do not put quotation marks around titles of articles or books. Capitalize only the first letter of books. Italicize journal or book titles, and volume numbers. Use " $\&$ " instead of "and" in multiple author listings.

10. Suggestions for style: Instead of "I drew a sample of 40" write "A sample of 40 was selected". Use "although" instead of "while", unless the meaning is "at the same time". Use "because" instead of "since", unless the meaning is "after". Instead of "Smith (1990) notes" write "Smith (1990) noted". Do not strike spacebar twice after a period.

\section{Print Subscriptions}

Print subscriptions including postage for professions is US \$60 per year; graduate students is US \$30 per year; and libraries, universities, and corporations is US \$195 per year. Subscribers outside of the US and Canada pay a US $\$ 10$ surcharge for additional postage. Online access is currently free at http://tbf.coe.wayne.edu/jmasm. Mail subscription requests with remittances to JMASM, P. O. Box 48023,

Oak Park, MI, 48237. Email journal correspondence, other than manuscript submissions, to jmasm@edstat.coe.wayne.edu.

\section{Notice To Advertisers}

Send requests for advertising information to jmasm@edstat.coe.wayne.edu. 


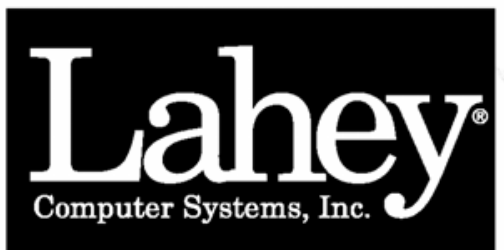

SOFTWARE SOLUTIONS

for Science \& Engineering

\section{Lahey/Fujitsu Fortran}

The standard for Fortran programming from the leader in Fortran language systems

LF95 Fortran for Linux and Windows

Full Fortran 95/90/77 support

Unsurpassed diagnostics

Intel and AMD optimizations
IMSL compatible

Fujitsu SSL2 math library

Wisk graphics package

\section{LF Fortran for the Microsoft ${ }^{\circledR}$.NET Framework - Coming Soon!}

Visual Studio integration

Windows / Web Forms designer

Project and code templates
On-line integrated help

XML Web services

ADO.NET support

Visit www.lahey.com for more information

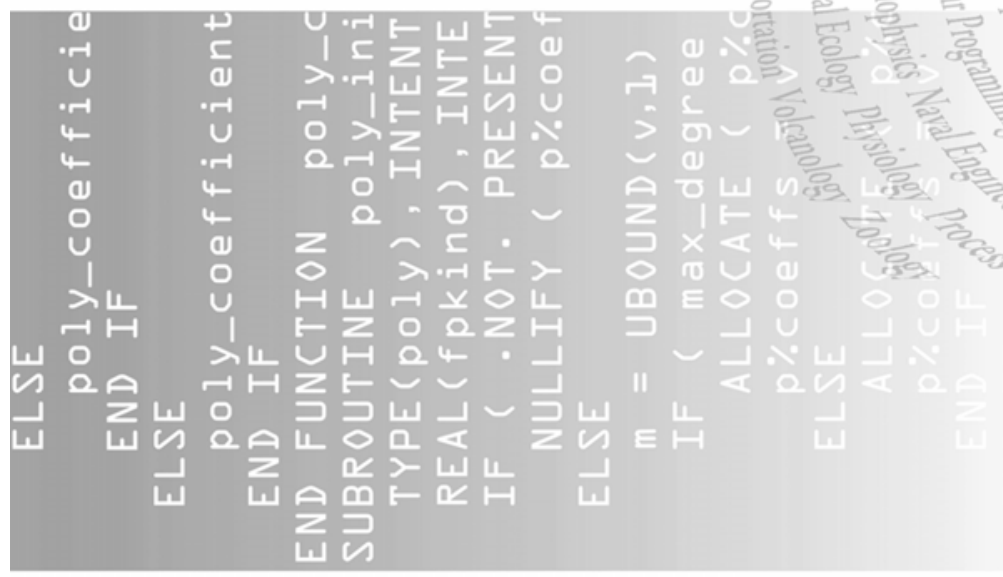

Lahey Computer Systems, Inc.

865 Tahoe Blvd - P.O. Box 6091

Incline Village, NV 89450 USA

1-775-831-2500

www.lahey.com

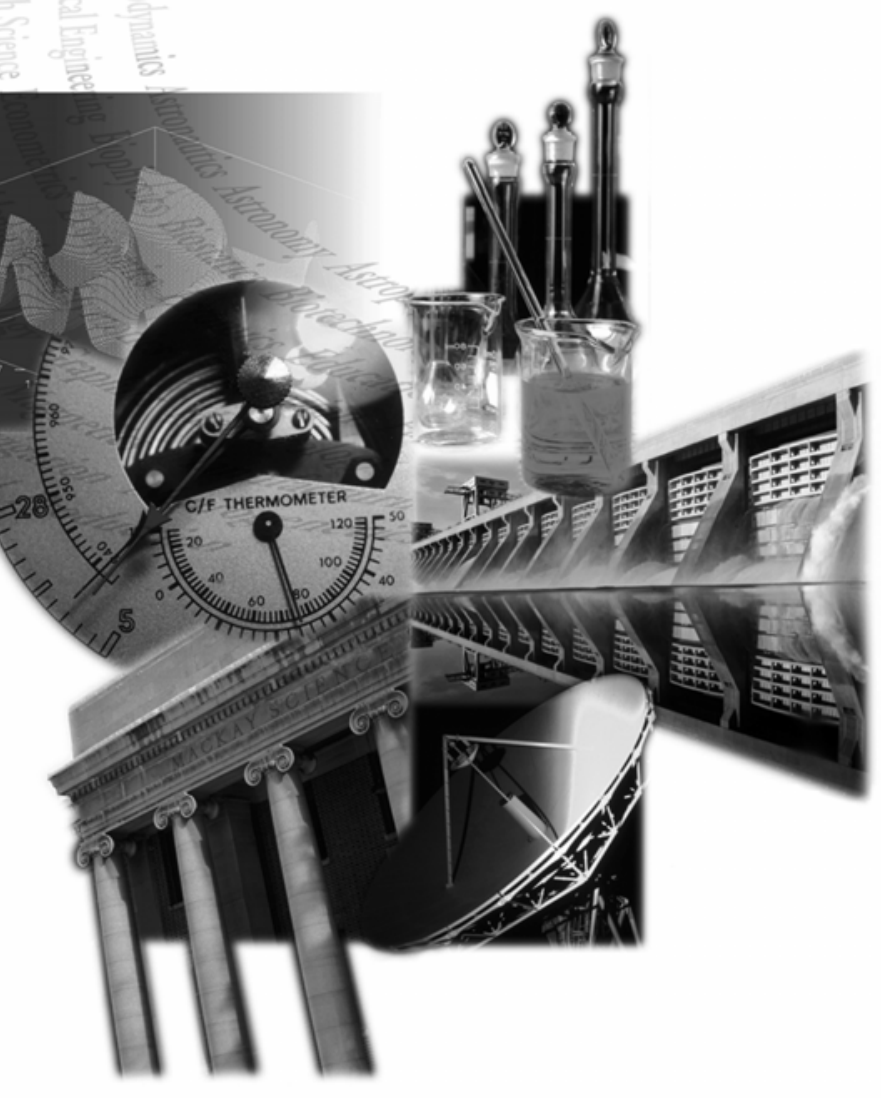




\section{Two Years in the Making...}

\section{Intel ${ }^{\oplus}$ Visual Fortran 8.0}

The next generation of Visual Fortran is here! Intel Visual Fortran 8.0 was developed jointly by Intel and the former DEC/Compaq Fortran engineering team.

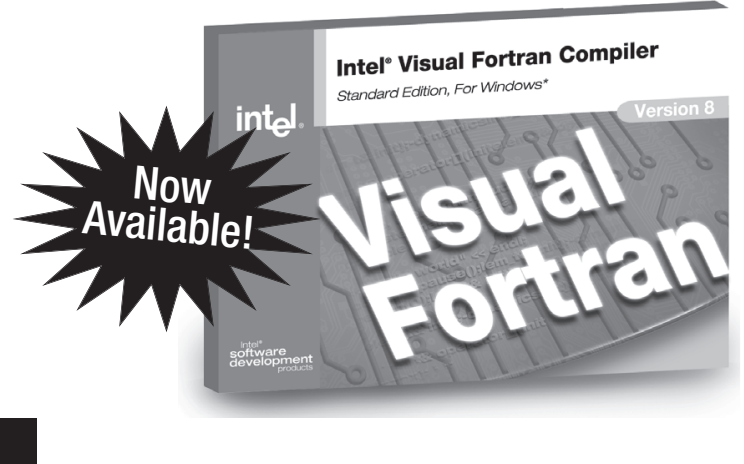

\section{Visual Fortran Timeline}

1997 DEC releases

Digital Visual Fortran 5.0

1998 Compaq acquires DEC and releases DVF 6.0

1999 Compaq ships CVF 6.1

2001 Compaq ships CVF 6.6

2001 Intel acquires CVF engineering team

2003 Intel releases

Intel Visual Fortran 8.0

Intel Visual Fortran $\mathbf{8 . 0}$

- CVF front-end + Intel back-end

- Better performance

- OpenMP Support

- Real*16

\section{Performance}

Outstanding performance on Intel architecture including Intel ${ }^{\circledR}$ Pentium $^{\circledR} 4$, Intel ${ }^{\circledR}$ Xeon $^{\mathrm{TM}}$ and Intel Itanium ${ }^{\circledR} 2$ processors, as well as support for Hyper-Threading Technology.

\section{Compatibility}

- Plugs into Microsoft Visual Studio* .NET

- Microsoft PowerStation4 language and library support

- Strong compatibility with Compaq* Visual Fortran

\section{Support}

1 year of free product upgrades and Intel Premier Support

"The Intel Fortran Compiler 7.0 was first-rate, and Intel Visual Fortran 8.0 is even better. Intel has made a giant leap forward in combining the best features of Compaq Visual Fortran and Intel Fortran. This compiler... continues to be a 'must-have' tool for any Twenty-First Century Fortran migration or software development project."

-Dr. Robert R. Trippi
Professor Computational Finance
University of California, San Diego

FREE trials available at: programmersparadise.com/intel

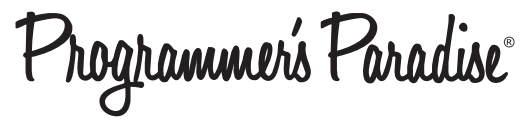

To order or request additional information call: 800-423-9990

Email: intel@programmers.com 